ISSN 0103-9954

\title{
CLASSIFICAÇÃO E CARACTERIZAÇ̃̃O DE ESTÁGIOS SUCESSIONAIS EM REMANESCENTES DE FLORESTA OMBRÓFILA MISTA NA FLONA DE SÃO FRANCISCO DE PAULA, RS, BRASIL
}

\author{
CLASSIFICATION AND CHARACTERIZATION OF STAGES OF SUCCESSION IN MIXED RAINY \\ FOREST REMAINS IN SÃO FRANCISCO DE PAULA'S NATIONAL FOREST (FLONA), RS, BRAZIL
}

\author{
Solon Jonas Longhi ${ }^{1}$ Doádi Antônio Brena ${ }^{2}$ Juliana Fernandes Gomes ${ }^{3}$ \\ Igor da Silva Narvaes ${ }^{4}$ Gabriel Berger ${ }^{5}$ Artur José Soligo ${ }^{6}$ \\ RESUMO
}

O presente trabalho integra o conjunto de estudos e pesquisas previstas no Projeto Ecológico de Longa Duração - PELD/CNPq, instalado FLONA de São Francisco de Paula, RS. Teve como objetivo identificar e caracterizar os diferentes estágios sucessionais de remanescentes de Floresta Ombrófila Mista. O trabalho foi realizado em dez conglomerados permanentes de $100 \mathrm{~m} \times 100 \mathrm{~m}\left(10.000 \mathrm{~m}^{2}\right)$, os quais foram divididos em dez faixas de $10 \mathrm{~m} \times 100 \mathrm{~m}\left(1.000 \mathrm{~m}^{2}\right)$ e estas subdivididas em dez subunidades de $10 \mathrm{~m} \times 10 \mathrm{~m}\left(100 \mathrm{~m}^{2}\right)$. Para a presente pesquisa, foi sorteada uma faixa de $10 \mathrm{~m} \times 100 \mathrm{~m}$ para cada conglomerado, totalizando cem subunidades amostrais (parcelas) onde foram instaladas, na altura do DAP, bandas (cintas) dendrométricas em todas as árvores com CAP $\geq 30 \mathrm{~cm}$, para medição anual dos diâmetros. A análise de agrupamento foi realizada por meio do programa Twinspan, utilizando uma matriz de dados com 99 parcelas (uma foi desconsiderada por não apresentar indivíduos com CAP $\geq 30 \mathrm{~cm}$ ) tendo como variável o incremento anual em diâmetro (obtido de 2 anos de observação) de 69 espécies. Obtiveram-se três grupos florísticos que indicaram estágios sucessionais na floresta: um grupo separou um estágio sucessional menos evoluído denominado de mata secundária, tendo Nectandra megapotamica e Cryptocarya aschersoniana como espécies indicadoras; um segundo grupo indicando um estágio sucessional intermediário da floresta, com Sebastiania commersoniana e Ocotea pulchella como espécies indicadoras; e um terceiro grupo constituído da floresta primária cuja espécie indicadora foi Araucaria angustifolia.

Palavras-chave: agrupamento; crescimento; manejo florestal.

\section{ABSTRACT}

The present work is part of studies and researches developed at Long Duration Ecological Project PELD/CNPq, located in São Francisco de Paula's National Forest (FLONA), RS. The objective of this paper was to identify and to characterize the different stages of succession in mixed rainy forest remains. The work had been accomplished in ten permanent conglomerates of $100 \mathrm{~m} \times 100 \mathrm{~m}\left(10.000 \mathrm{~m}^{2}\right)$, which were divided into ten strips of $10 \mathrm{~m} \times 100 \mathrm{~m}\left(1.000 \mathrm{~m}^{2}\right)$ and these ones subdivided into ten subunits of $10 \mathrm{~m} \times 10 \mathrm{~m}\left(100 \mathrm{~m}^{2}\right)$. In this research, a strip of $10 \mathrm{~m} \times 100 \mathrm{~m}$ was selected for each conglomerate, totaling 100 subunits (stands), where it had been installed, at DBH height, dendrometric bands in all trees with $\mathrm{CBH} \geq 30 \mathrm{~cm}$, for annual diameters measurement. The cluster analysis was accomplished through the Twinspan program, using a data matrix with ninety-nine stands (one of those was unconsidered by the absence of individuals with $\mathrm{CBH} \geq$ $30 \mathrm{~cm}$ ) having as variable the annual increment in diameter (obtained in two years of observations) of sixty nine species. Three floristic groups was obtained, indicating stages of succession in the forest: a group separated a less developed stage of succession denominated secondary forest, being Nectandra megapotamica and Cryptocaria aschersoniana the indicative species; a second group indicated an

1. Engenheiro Florestal, Dr., Professor Titular do Departamento de Ciências, Centro de Ciências Rurais, Universidade Federal de Santa Maria, CEP: 97105-900, Santa Maria (RS). sjlonghi@smail.ufsm.br

2. Engenheiro Florestal, Dr., Professor Adjunto do Departamento de Ciências, Centro de Ciências Rurais, Universidade Federal de Santa Maria, CEP: 97105-900, Santa Maria (RS).dabrena@via-rs.net

3. Engenheira Florestal, M.Sc., Analista de Inventário Florestal, Setor de Planejamento Florestal. Aracruz Celulose S/A, Rua São Geraldo, 1680, Bairro Ermo, CEP: 92500-000, Guaiba (RS).jfgomes@aracruz.com.br

4. Engenheiro Florestal, M.Sc., CEP: 97050-480, Santa Maria (RS). igornarvaes@gmail.com.br

5. Engenheiro Florestal, Mestrando em Engenharia Florestal, Programa de Pós-Graduação em Engenharia Florestal, Universidade Federal de Santa Maria, CEP: 97105-900, Santa Maria (RS).

6. Engenheiro Florestal, Esp. em Planejamento Florestal, Diretor da FLONA de São Francisco de Paula, RS-484, Km 6, Rincão dos Kroeff, CEP: 95400-000, São Francisco de Paula (RS). asoligo@ibest.com.br

Recebido para publicação em 26/04/2005 e aceito em 16/11/2005. 
intermediate stage of succession in the forest, being Sebastiania commersoniana and Ocotea pulchella the indicative species; and a third group of primary forest, whose indicative species was Araucaria angustifolia.

Keywords: cluster; growth; forest management.

\section{INTRODUÇÃO}

Este trabalho integra o conjunto de estudos e pesquisas previstas no Projeto Ecológico de Longa Duração - PELD/CNPq - "Conservação e Manejo Sustentável de Ecossistemas Florestais - Bioma Araucária e suas Transições", em andamento na Unidade Experimental de Pesquisa: Floresta Nacional de São Francisco de Paula e Fazenda Tupi, Nova Prata, RS. Visa a caracterizar detalhada e integradamente os processos, mecanismos e fatores relevantes no Bioma Floresta de Araucária e suas Transições, tendo como objetivo principal "Quantificar e qualificar, em longo prazo, o grau de alteração na produtividade e biodiversidade deste Bioma em razão da magnitude e da intensidade das atividades antrópicas”.

No presente estudo, procurou-se identificar e caracterizar os diferentes estágios sucessionais ocorrentes ao longo do processo de dinâmica da floresta. Estudos dessa natureza em florestas tropicais têm sido intensificados nas últimas décadas, não apenas com relação à descrição da sua composição florística e estrutura fitossociológica, mas também buscando entender a dinâmica desses ecossistemas. Um dos aspectos dinâmicos que se tem pesquisado é a sucessão florestal (Gandolfi, 1991).

Horn (1974) define sucessão ecológica como um fenômeno que envolve gradativas variações na composição específica e na estrutura da comunidade, iniciando-se o processo em áreas que, mediante ações perturbatórias ou não, se apresentam disponíveis à colonização de plantas e animais, prosseguindo até determinado período onde tais mudanças se tornam bastante lentas, sendo a comunidade resultante designada como clímax.

Odum (1988) salienta que, quando a sucessão não é interrompida por forças externas, é bastante direcional e previsível; envolvendo modificação do ambiente físico pelos fatores bióticos, no sentido de aumentar a complexidade estrutural e atingir um grau máximo de biomassa e de função simbiótica entre organismos por unidade de fluxo energético disponível.

Com relação ao conceito ecológico de sucessão, Gómez-Pompa \& Wiechers (1976) comentam que uma das características universais de todo o ecossistema é a troca contínua a que está submetido.

A seqüência inteira de comunidades que se substituem uma às outras numa determinada área chamase sere; as comunidades relativamente transitórias são chamadas de estágios serais (ou subseres); o sistema estabilizado terminal e autoperpetuante é denominado de clímax. Quando o processo de formação de uma comunidade se dá sobre um substrato parcialmente desocupado, é denominado sucessão primária, enquanto que aquele que começa num local anteriormente ocupado por uma comunidade é denominado sucessão secundária (Odum, 1988).

A sucessão florestal sempre inicia com etapas pioneiras e culmina com a de clímax. Conforme Daubenmire (1968), a progressão de uma sere acarreta: mudanças na dominância de plantas de pequeno porte e de posições inferiores na escala filogenética por plantas grandes, no alto desta escala; aumento na longevidade das dominantes; convergência para um tipo físionômico prevalecente e característico da região; diversificação das formas de vida; substituição de espécies com amplitudes ecológicas similares e amplas, por grupos com limites estreitos e necessidades complementares; aumento do número de dependências interespecíficas; aumento na massa de tecidos vivos e de matéria orgânica morta por unidade de área; aumento na regularidade da composição florística e estrutura entre os grupos que representam uma associação; aumento do número de possíveis "patamares" ao longo dos quais a matéria circula e a energia flui; amenização dos extremos microambientais; maturação do perfil do solo; maior resistência do ecossistema a distúrbios externos.

A compartimentalização do processo de sucessão secundária em fases ou estágios sucessionais distintos, mesmo arbitrários, é um artifício utilizado na busca do entendimento sobre a dinâmica funcional das florestas (Kageyama et al., 1986).

Dessa forma, Klein (1979 e 1980), no estudo da ecologia do Vale do Itajaí/SC, caracterizou a dinâmica da Floresta Ombrófila Densa, especialmente em relação a períodos subseqüentes à ação antrópica 
sobre a comunidade. Demonstrou que o processo de regeneração dessa floresta está intimamente associado ao grau de degradação das condições locais e caracterizou a diversidade vegetal de espécies em estágios de sucessão secundária classificados como: estágios pioneiros, capoeirinha, capoeira, capoeirão e floresta secundária.

De acordo com Vaccaro (1997), o que se observa, na germinação, estabelecimento, desenvolvimento e reprodução de espécies florestais, é a existência de um grande espectro de variação nas respostas apresentadas a esses processos em razão da intensidade luminosa presente no sítio. Assim, encontram-se de um lado espécies que dependem de luminosidade e temperatura para sua germinação, estabelecimento, desenvolvimento e reprodução. No extremo oposto deste espectro de respostas, situam-se espécies que não suportam as condições de plena exposição a altas intensidades luminosas e de temperatura, necessitando germinarem e desenvolverem-se à sombra de outras árvores. Entre os dois extremos, entretanto, existe um grande número de espécies que apresentam características ou adaptações ecológicas intermediárias, quanto às exigências e tolerâncias à luz, variando também em relação ao aspecto considerado, seja a germinação, o estabelecimento, o desenvolvimento ou a reprodução.

Dessa maneira, muitos autores têm agrupado as espécies florestais em grupos ecológicos com base na estratégia de regeneração que cada uma apresenta.

Budowski (1965) apresentou um modelo para as florestas tropicais em que a sucessão secundária é formada por um conjunto de estágios sucessionais distintos e as espécies, por sua vez, são agrupadas em função de sua ocorrência preferencial em cada um destes estágios. Nesse modelo, aponta a conveniência de denominar os estágios serais em pioneiro, secundário inicial, secundário tardio e clímax.

Rodrigues (1995) comenta que as espécies pioneiras têm função cicatrizadora de ambientes perturbados. No outro extremo das pioneiras, têm-se as clímax que são as espécies finais na substituição seqüencial de espécies na sucessão. Entre os dois extremos, existe um grande número de espécies com características ou adaptações ecológicas intermediárias. Quando as características são mais parecidas com as pioneiras, estas espécies são chamadas de secundárias iniciais; quando apresentam características mais próximas das espécies clímax, são denominadas secundárias tardias.

Para Whitmore (1989), as espécies tropicais pertencem somente a dois grandes grupos ecológicos, sendo um grupo formado pelas espécies intolerantes à sombra e outro formado pelas espécies tolerantes à sombra. As demais variações, que eventualmente se observam, estariam abrangidas por toda essa dicotomia.

São consideradas espécies intolerantes à sombra aquelas incapazes de se desenvolverem sob o dossel da floresta madura, necessitando, assim, de clareiras ou fases sucessionais da floresta onde as condições de luz sejam propícias. Espécies tolerantes à sombra são aquelas capazes de se desenvolverem sob o dossel da floresta até atingirem o estágio reprodutivo no qual necessitam condições mais adequadas de luminosidade.

De acordo com Rodrigues (1995), a classificação sucessional de espécies florestais tem sido um ponto muito polêmico em estudos de florestas tropicais, pois pouco se conhece da autoecologia das espécies, que forneceria os dados necessários para sua classificação mais adequada. Essa classificação das espécies auxiliaria na compreensão da dinâmica florestal e na adoção de práticas adequadas de manejo e recuperação de florestas.

Quanto aos métodos de estudo da sucessão secundária em ecossistemas florestais, Gómez-Pompa \& Wiechers (1976) comentam que se pode seguir vários caminhos: um primeiro método consiste em estudar através do tempo o que se sucede em uma área determinada após esta ter sido perturbada; porém, esse método apresenta sérias limitações, já que requer um espaço de tempo demasiado grande para se obterem resultados sobre o processo geral de sucessão. Outro método consiste em estudar, em uma mesma zona ecológica, diversos estágios sucessionais de idade conhecida; pois, com base na informação obtida por tais amostras no espaço, podem-se interpretar as trocas no tempo. Outra maneira de abordar a sucessão é buscando informações biológicas para poder interpretar, em relação ao tempo, os possíveis mecanismos dos processos de regeneração.

Com relação ao método de comparação de áreas de diferentes estágios sucessionais, Kuniyoshi (1989) confirma que o tipo de clima e solo das áreas deve ser o mesmo; e ressalta a importância de se conhecer ecologicamente muito bem as áreas de estudo, pois comunidades diferentes podem ser interpretadas 
como uma cronoseqüência.

Assim, o presente trabalho teve como objetivo classificar e caracterizar remanescentes de Floresta Ombrófila Mista na FLONA de São Francisco de Paula em grupos florísticos representativos dos estágios sucessionais ocorrentes ao longo da dinâmica da floresta, visando a entender sua estrutura, composição e dinâmica, fatores fundamentais para desenvolvimento de planos de manejo florestal sustentável.

\section{MATERIAL E MÉTODOS}

\section{Caracterização da área de estudo}

A área onde foi realizado o estudo se localiza na Floresta Nacional de São Francisco de Paula (FLONA), município de São Francisco de Paula no estado do Rio Grande do Sul, a $27 \mathrm{Km}$ da cidade, entre as coordenadas geográficas $29^{0} 02^{\prime}$ de latitude sul e $50^{0} 23^{\prime}$ longitude oeste.

O clima da região é temperado com invernos rigorosos, registrando-se neve em algumas ocasiões. Há ocorrência de geadas e nevoeiros. A temperatura média anual é inferior a $18,5^{\circ} \mathrm{C}$ (Moreno, 1961).

A área da FLONA caracteriza-se por ter um relevo ondulado a fortemente ondulado com recorte profundo de alguns rios. Há regiões abruptas que originam um relevo relativamente movimentado. A vegetação natural pertence ao tipo fitogeográfico Floresta Ombrófila Mista ou Mata de Araucária ou de Pinheiro brasileiro, cobrindo cerca de $56 \%$ de sua área total.

\section{Método de amostragem}

O trabalho foi realizado em dez conglomerados permanentes de $100 \mathrm{~m} \times 100 \mathrm{~m}\left(10.000 \mathrm{~m}^{2}\right)$, os quais foram divididos em dez faixas de $10 \mathrm{~m} \times 100 \mathrm{~m}\left(1.000 \mathrm{~m}^{2}\right)$ e estas subdivididas em dez subunidades (parcelas) de $10 \mathrm{~m} \times 10 \mathrm{~m}\left(100 \mathrm{~m}^{2}\right)$, conforme mostra a Figura 1 .

Para a presente pesquisa, foi sorteada uma faixa de $10 \mathrm{~m} \times 100 \mathrm{~m}$ para cada conglomerado, totalizando dez faixas ou cem unidades amostrais de $10 \mathrm{~m} \times 10 \mathrm{~m}$ onde foram instaladas, na altura do DAP, bandas dendrométricas em todas as árvores com CAP $\geq 30 \mathrm{~cm}$, para medição dos incrementos médio anual em diâmetro $(\mathrm{cm})$. Uma subunidade amostral (parcela) não apresentou indivíduos com $\mathrm{CAP} \geq 30 \mathrm{~cm}$ sendo, portanto, eliminada da análise. No total, a amostragem ficou constituída de 99 parcelas, onde foram realizadas quatro medições anuais, uma em cada estação climática (primavera, verão, outono e inverno) durante um período de 2 anos de observação.

Após a instalação das bandas dendrométricas, esperaram-se duas estações de crescimento (aproximadamente 6 meses) para haver um perfeito ajustamento delas ao tronco das árvores, momento do início da validação dos dados.

As bandas dendrométricas foram construídas utilizando-se chapas de alumínio de $2 \mathrm{~mm}$ de espessura e $2,5 \mathrm{~cm}$ de largura, graduadas em $\mathrm{mm}$.

Todas as árvores contempladas com as bandas dendrométicas foram identificadas e medidas suas respectivas circunferências à altura do peito (CAP).

A localização e instalação das parcelas em campo foram realizadas com o auxílio do GPS (Global Positioning System), o qual precisará a localização de cada unidade e subunidade amostral. As parcelas foram instaladas com teodolito ou esquadro de agrimensor, marcando-se os limites externos, as faixas e as subunidades com canos de PVC rígido.

A identificação de todos os indivíduos amostrados possibilitou o estudo da composição florística da área de estudo. Das espécies que não foram identificadas in loco foi coletado material botânico fértil ou não, e levado para posterior identificação junto ao Herbário do Departamento de Ciências Florestais (HDCF) ou enviado a especialistas. 


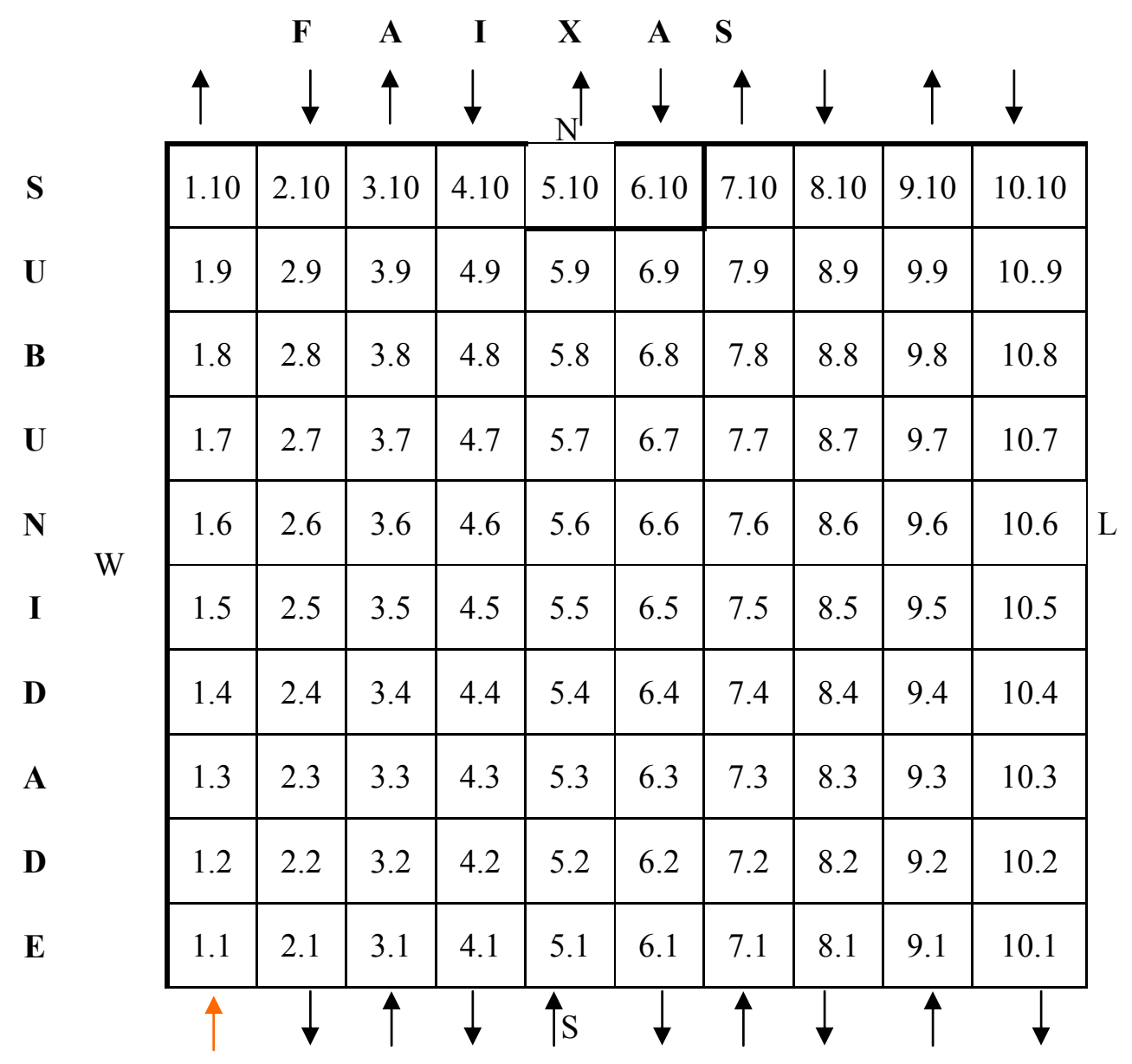

FIGURA 1: Desenho esquemático da unidade amostral permanente de 1 ha $(100 \mathrm{~m} \times 100 \mathrm{~m})$ e suas subunidades $(10 \mathrm{~m} \times 10 \mathrm{~m})$ para o levantamento do incremento médio anual na Floresta Ombrófila Mista na FLONA de São Francisco de Paula, RS, Brasil.

FIGURE 1: Scheme figure of the permanent sample unit of 1 ha $(100 \mathrm{~m} \times 100 \mathrm{~m})$ and its subunits $(10 \mathrm{~m} \times$ $10 \mathrm{~m})$ to the survey of mean annual increment in the mixed rainy forest at FLONA in São Francisco de Paula, RS, Brazil.

\section{Método de análise}

A identificação dos estágios sucessionais na Floresta Ombrófila Mista da FLONA de São Francisco de Paula foi feita pela análise de agrupamento utilizando o método de classificação Twinspan (Two-way Indicator Species Analysis), o qual verifica a ocorrência de padrões na distribuição de espécies, associados às condições ambientais locais constatadas no campo, nesse caso, considerando o crescimento (Hill, 1979). Para isso, foi elaborada uma matriz de dados com 99 parcelas (uma foi desconsiderada por não apresentar indivíduos com CAP $\geq 30 \mathrm{~cm}$ ) tendo como variáveis o incremento anual em diâmetro (obtido de 2 anos de observação) de 69 espécies amostradas.

Para definição dos agrupamentos, além da análise estatística (multivariada), levaram-se em consideração as características observadas em campo como possível explicação para a formação de tais grupos. Conforme Kent e Coker (1992), o número de subdivisões depende do tamanho e característica do conjunto de dados, considerando que não necessariamente todas as divisões são definidas como um grupo, mas aquelas que podem ser verificadas in loco e explicadas. Se a divisão produzir subgrupos que não refletem as características da floresta, estes devem, então, ser mesclados. Conforme Araujo (2002), para as divisões serem relevantes devem ter um autovalor maior ou igual a 0,30 (30\% da variância total dos dados).

O resultado final do Twinspan é a descrição das divisões realizadas, com respectivos autovalores, unidades amostrais que pertencem a cada grupo, espécies indicadoras e preferenciais, amostras 
malclassificadas na linha divisória de cada agrupamento, além de uma matriz organizada com a classificação hierárquica das amostras e espécies em conjunto.

Os diferentes estágios sucessionais encontrados foram caracterizados pela análise da composição florística das espécies e da análise do crescimento das mesmas.

\section{RESULTADOS E DISCUSSÃO}

\section{Análise de agrupamento}

A Análise de Cluster (Twinspan) definiu três grupos florísticos distintos entre as parcelas avaliadas (Figura 2), os quais se diferenciaram em relação às suas características ecológicas que foram determinadas por meio do incremento médio anual em diâmetro das espécies. Observa-se que a análise relaciona as espécies indicadoras de cada grupo, sendo espécies com preferências ecológicas que podem identificar as condições ambientais das subunidades (Hill et al., 1975).

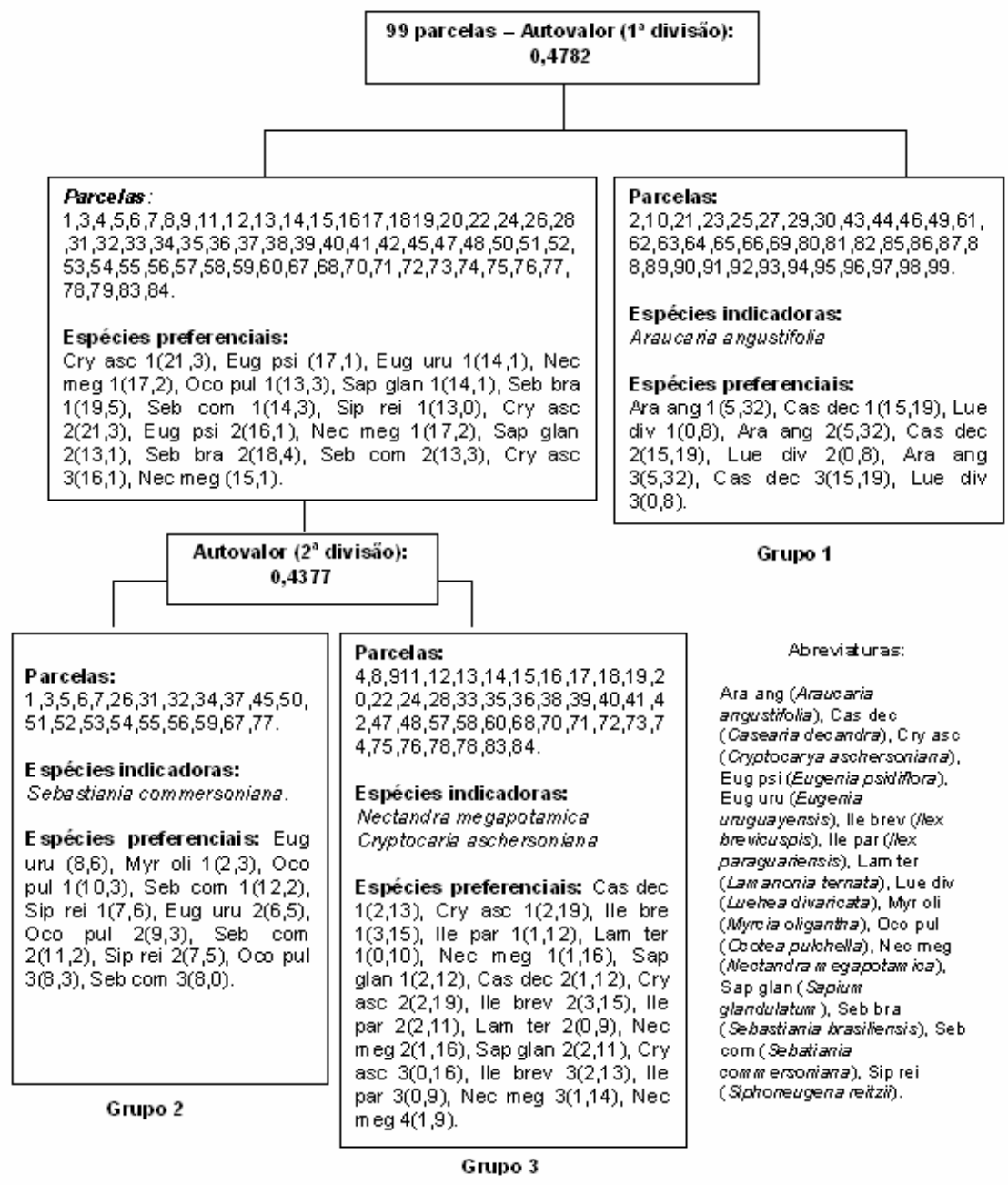

FIGURA 2: Classificação das uniudades amostrais em três grupos florísticos em Floresta Ombrófila Mista da FLONA de São Francisco de Paula, RS, Brasil.

FIGURE 2: Classification of sample units in three floristic groups in the Mixed Rainy Forest at FLONA in São Francisco de Paula, RS, Brazil.

A primeira divisão das subunidades amostrais formou dois grandes grupos. O AUTOVALOR de 0,4782 $(>0,30)$ mostrou que houve diferenciação entre eles. Essa primeira divisão mostrou que existe similaridade florística entre as parcelas relacionadas na esquerda da Figura 2 que, por sua vez, são diferentes das relacionadas na direita que também são similares entre si. A maioria das espécies não se mostraram exclusivas, porém ocorrem com maior incremento em determinado grupo de acordo com suas preferências 
ecológicas. A Araucaria angustifolia foi a espécie indicadora do grupo da direita (positivo) tendo Casearia decandra e Luehea divaricata como espécies preferenciais. Pelas características das espécies, constitui o grupo mais representativo do estágio clímax da floresta ou o grupo mais característico da Floresta Ombrófila Mista da área.

O grupo da esquerda (negativo) apresentou Cryptocarya aschersoniana, Eugenia psidiiflora, Eugenia uruguayensis, Nectandra megapotamica, Ocotea pulchella, Sapium glandulatum, Sebastiania brasiliensis, Sebastiania commersoniana e Siphoneugena reitzii como espécies preferenciais. Da mesma forma, pelas características das espécies, constitui o grupo das formações secundárias da floresta, de áreas mais abertas e com solos mais úmidos. A não-existência de espécie indicadora neste grupo, provavelmente, se deve ao fato da ocorrência de um grande número de espécies tolerantes a locais úmidos.

$\mathrm{Na}$ segunda divisão, com um AUTOVALOR 0,4377 $(>0,30)$, separou o primeiro agrupamento da esquerda em dois grupos. Um grupo menor tendo Sebastiania commersoniana e Ocotea pulchella como espécies indicadoras, Eugenia uruguayensis, Myrcia oligantha e Siphoneugena reitzii como espécies preferenciais; um grupo maior à direita, tendo Nectandra megapotamica e Cryptocaria aschersoniana como espécies indicadoras; e Casearia decandra, Ilex brevicuspis, Ilex paraguariensis, Lamanonia ternata e Sapium glandulatum como espécies preferenciais.

$\mathrm{Na}$ terceira divisão, no agrupamento à direita, com AUTOVALOR 0,4843, geraram dois grupos similares do ponto de vista florístico, um deles com poucas parcelas. Embora o autovalor da divisão tenha sido $>0,30$, considerou-se como um grupo distinto. Segundo Hill (1979), fica a critério do pesquisador a escolha do número de grupos, caso as divisões ainda continuem atingindo autovalores superiores a 0,30.

Os agrupamentos formados geraram novas divisões, porém, pelas observações de campo e pelos objetivos desejados no estudo, considerou-se a diferenciação de três grupos florísticos distintos que demonstraram claramente as diferenças em crescimento e preferências ecológicas da área. Foram, de acordo com as características sucessionais das espécies indicadoras e preferenciais, assim denominados:

Grupo 1 - Floresta de locais úmidos;

Grupo 2 - Floresta secundária;

Grupo 3 - Floresta primária.

Conforme a Figura 2, o número após a espécie se refere a pseudoespécies (nível de corte), enquanto que o número entre parênteses se refere às parcelas onde estão contidas as pseudoespécies em ambos lados da divisão.

\section{Caracterização geral dos grupos}

A listagem das espécies amostradas na floresta, com seus respectivos nomes científicos, famílias e nomes vulgares, pode ser observada no Tabela 1.

No Grupo 1 (floresta de locais úmidos), foram encontradas 35 espécies pertencentes a 27 gêneros e 16 famílias botânicas, enquanto que no Grupo 2 (floresta secundária) foram encontradas 49 espécies pertencentes a 38 gêneros e 21 famílias botânicas e, no Grupo 3 (floresta primária) 47 espécies pertencentes a 36 gêneros e 22 famílias botânicas. Em todos os grupos, as famílias Myrtaceae e Lauraceae foram as mais representativas. Tal situação é comum nas florestas e em todos os tipos fitogeográficos do Rio Grande do Sul, conforme trabalhos de Jarenkow (1985), Longhi (1997), Vaccaro (1997), Rio Grande do Sul (2002), Narvaes (2004), entre outros. Destacaram-se também as famílias Euphorbiaceae nos três grupos e Flacourtiaceae no Grupo 3. 
TABELA 1: Espécies amostradas na área de estudo da Floresta Ombrófila Mista da FLONA de São Francisco de Paula, RS, com os respectivos nomes científicos, famílias e nomes populares.

TABLE 1: Sampled species in the sampling area of mixed rainy forest at FLONA in São Francisco de Paula, RS, with their scientific names, families and popular names.

\begin{tabular}{|c|c|c|}
\hline Nome Científico & Família & Nome Popular \\
\hline Lithraea brasiliensis Marchand & Anacardiaceae & Aroeira-bugre \\
\hline Rollinia rugulosa Schltdl. & Annonaceae & Araticum-quaresma \\
\hline Ilex brevicuspis Reissek & Aquifoliaceae & Caúna-da-serra \\
\hline Ilex dumosa Reissek & Aquifoliaceae & Congonha \\
\hline Ilex paraguariensis A. St.-Hil. & Aquifoliaceae & Erva-mate \\
\hline Araucaria angustifolia (Bertol.) Kuntze & Araucariaceae & Pinheiro-brasileiro \\
\hline Dasyphyllum spinescens (Less.) Cabrera & Asteraceae & Açucará \\
\hline Dasyphyllum tomentosum (Spreng.) Cabrera & Asteraceae & Açucará-piloso \\
\hline Vernonia discolor (Spreng.) Less. & Asteraceae & Vassourão-preto \\
\hline Maytenus evonymoides Reissek & Celastraceae & Coração-de-bugre \\
\hline Lamonia ternata Vell. & Cunoniaceae & Guaperê \\
\hline Dicksonia sellowiana Hook. & Dicksoniaceae & Xaxim \\
\hline Sapium glanutum (Vell.) Pax. & Euphorbiaceae & Pau-leiteiro \\
\hline Sebastiania brasiliensis Spreng. & Euphorbiaceae & Branquilho-leiteiro \\
\hline Sebastiania commersoniania (Baill.) L. B. Sm. et Downs & Euphorbiaceae & Branquilho \\
\hline Lonchocarpus campestris Mart. ex Benth. & Fabaceae & Pau-canzil \\
\hline Banara parviflora (A. Gray) Benth. & Flacourtiaceae & Guaçatunga-preta \\
\hline Casearia decandra Jacq. & Flacourtiaceae & Guaçatunga \\
\hline Casearia obliqua Spreng. & Flacourtiaceae & Estralador \\
\hline Xylosma pseudosalzmannii Sleumer & Flacourtiaceae & Espinho-de-judeu \\
\hline Xylosma tweedianum (Clos) Eichler & Flacourtiaceae & Sucará \\
\hline Citronella gongonha (Mart.) Howard & Icacinaceae & Gongonha-espinhosa \\
\hline Cinnamomum amoenum (Nees) Kosterm. & Lauraceae & Canela \\
\hline Cinnamomum glaziovii (Mez) Kosterm. & Lauraceae & Canela \\
\hline Cryptocarya aschersoniania $\mathrm{Mez}$ & Lauraceae & Canela-vick \\
\hline Cryptocarya moschata Nees & Lauraceae & Canela-cega \\
\hline Nectandra megapotamica (Spreng.) Nez & Lauraceae & Canela-preta \\
\hline Ocotea indecora (Schott) Mez & Lauraceae & Canela \\
\hline Ocotea puberula (A. Rich.) Nees & Lauraceae & Canela-guaicá \\
\hline Ocotea pulchella Mart. & Lauraceae & Canela-langeana \\
\hline Cedrela fissilis Vell. & Meliaceae & Cedro \\
\hline Acacia bonariensis Gill. ex Hook. et Arn. & Mimosaceae & Unha-de-gato \\
\hline Inga vera Willd. & Mimosaceae & Ingá-banana \\
\hline Myrsine umbelata Mart. & Myrsinaceae & Capororocão \\
\hline Myrsine sp. & Myrsinaceae & Capororoca \\
\hline Acca sellowiana (O. Berg) Burret & Myrtaceae & Goiaba-serrana \\
\hline Blepharocalyx salicifolius (Kunth) O. Berg & Myrtaceae & Murta \\
\hline Calyptranthes concinna DC. & Myrtaceae & Guamirim-de-facho \\
\hline Campomanesia rhombea O. Berg & Myrtaceae & Guabiroba-miúda \\
\hline Campomanesia xanthocarpa O. Berg & Myrtaceae & Guabiroba \\
\hline Eugenia involucrata DC. & Myrtaceae & Cerejeira-do-mato \\
\hline Eugenia psidiiflora $\mathrm{O}$. Berg & Myrtaceae & Guamirim \\
\hline Eugenia uruguayensis Cambess. & Myrtaceae & Batinga-vermelha \\
\hline Myrceugenia cucullata D. Legrand & Myrtaceae & Guamirim-quebradiço \\
\hline
\end{tabular}


TABELA 1: Continuação ...

TABLE 1: Continued ...

\begin{tabular}{llll}
\hline \multicolumn{1}{c}{ Nome Científico } & Família & Nome Popular \\
\hline Myrceugenia miersiana (Gardner) D. Legrand et Kausel & Myrtaceae & Guamirim-piloso \\
Myrcia oligantha O. Berg & Myrtaceae & Guamirim \\
Myrcianthes gigantea (D. Legrand) D. Legrand & Myrtaceae & Araça-do-mato \\
Myrcianthes pungens (O. Berg) D. Legrand & Myrtaceae & Guabijú \\
Myrciaria floribunda (West ex Willd.) O. Berg & Myrtaceae & Camboim \\
Myrrhinium atropurpureum Schott & Myrtaceae & Murtilho \\
Siphoneugena reitzii D. Legrand & Myrtaceae & Camboim-de-reitz \\
Seguieria aculeata L. & Phytolaccaceae & Cipó-umbú \\
Podocarpus lambertii Klotzsch ex Endl. & Podocarpaceae & Pinheiro-bravo \\
Roupala brasiliensis Klotzsch & Proteaceae & Carvalho-brasileiro \\
Scutia buxifolia Reissek & Rhamnaceae & Coronilha \\
Prunus myrtifolia (L.) Urb. & Rosaceae & Pessegueiro-do-mato \\
Policarpus pennatifolius Lem. & Rutaceae & Cutia \\
Zanthoxylum petiolare A. St.-Hil. et Tul. & Rutaceae & Mamicão \\
Zanthoxylum rhoifolium L. & Rutaceae & Mamica-de-cadela \\
Cupania vernalis Cambess. & Sapindaceae & Camboáta-vermelho \\
Matayba elaeagnoides Radlk. & Sapindaceae & Camboáta-branco \\
Solanum mauritianum Scop. & Solanaceae & Fumo-bravo \\
Solanum sanctae-catharinae Dunal & Solanaceae & Joá-manso \\
Symplocos tetrandra (Mart.) Miq. & Symplocaceae & Sete-sangrias \\
Symplocos uniflora (Pohl) Benth. & Symplocaceae & Sete-sangrias \\
Gordonia acutifolia (Wawra) H. Keng & Theaceae & Santa-rita \\
Luehea divaricata Mart. et Zucc. & Tiliaceae & Açoita-cavalo \\
Celtis iguanaea (Jacq.) Sargent & Ulmaceae & Esporão-de-galo \\
Cipós & - & - \\
\hline
\end{tabular}

Observou-se que os Grupos 2 e 3 apresentaram maior riqueza florística. O Grupo 3 por ser o grupo de floresta primária, em estado clímax, constituindo o grupo da formação típica de Floresta Ombrófila Mista. O Grupo 2 por ser o grupo de formação secundária já em adiantado estágio de desenvolvimento, ainda com muitas espécies pioneiras e já com espécies da floresta primária.

O Grupo 1, de menor riqueza florística, é o grupo em estágio intermediário de sucessão e localiza-se em áreas baixas e planas, com solos úmidos.

No Grupo 1, das áreas mais úmidas, predominam as espécies Sebastiania commersoniana, Eugenia psidiiflora, Ocotea pulchella, Eugenia uruguayensis e Myrcia oligantha, além de Myrciaria floribunda, Ocotea indecora, Sebastiania brasiliensis, Roupala brasiliensis, Scutia buxifolia e Siphonengena reitzii. Foram exclusivas deste grupo às espécies Acácia bonariensis, Celtis iguanaea e Seguieria aculeata (Tabela 2).

TABELA 2: Número de indivíduos e incremento médio anual em diâmetro $(\mathrm{cm})$ das espécies encontradas nos respectivos grupos determinados.

TABLE 2: Number of individuals and mean annual increment in diameter $(\mathrm{cm})$ of found species in determined groups.

\begin{tabular}{l|cc|c|c|c|c|c|c|c}
\hline \multirow{2}{*}{ Espécie } & \multicolumn{2}{|c|}{ Grupo 1 } & \multicolumn{2}{c|}{ Grupo 2 } & \multicolumn{2}{c|}{ Grupo 3 } & \multicolumn{2}{c}{ Total } \\
\cline { 2 - 10 } & $\mathrm{N}$ & $\mathrm{I}$ & $\mathrm{N}$ & $\mathrm{I}$ & $\mathrm{N}$ & $\mathrm{I}$ & $\mathrm{N}$ & $\mathrm{I}$ \\
\hline Acácia bonariensis & 1 & 0,38 & & & & & 1 & 0,38 \\
Acca sellowiana & & & 1 & 0,10 & & & 1 & 0,10 \\
Araucaria angustifolia & 1 & 0,25 & 8 & 0,59 & 89 & 0,54 & 98 & 0,46 \\
\hline & & & & & & \multicolumn{4}{r}{ Continua ... }
\end{tabular}


TABELA 2: Continuação ...

TABLE 2: Continued ...

\begin{tabular}{|c|c|c|c|c|c|c|c|c|}
\hline \multirow[t]{2}{*}{ Espécie } & \multicolumn{2}{|c|}{ Grupo 1} & \multicolumn{2}{|c|}{ Grupo 2} & \multicolumn{2}{|c|}{ Grupo 3} & \multicolumn{2}{|c|}{ Total } \\
\hline & $\mathrm{N}$ & I & $\mathrm{N}$ & I & $\mathrm{N}$ & I & $\mathrm{N}$ & I \\
\hline Banara parviflora & & & & & 1 & 0,92 & 1 & 0,92 \\
\hline Blepharocalyx salicifoius & 7 & 1,01 & 12 & 0,80 & 22 & 0,57 & 41 & 0,79 \\
\hline Calyptranthes concinna & 3 & 0,38 & 4 & 0,33 & & & 7 & 0,36 \\
\hline Campomanesia rhombea & 5 & 0,38 & 8 & 0,42 & 4 & 0,80 & 17 & 0,53 \\
\hline Campomanesia xanthocarpa & 1 & 0,65 & 10 & 0,79 & 4 & 0,68 & 15 & 0,71 \\
\hline Casearia decandra & 2 & 0,65 & 13 & 0,58 & 26 & 0,79 & 41 & 0,67 \\
\hline Casearia obliqua & & & & & 4 & 0,80 & 4 & 0,80 \\
\hline Cedrela fissilis & & & & & 1 & 1,72 & 1 & 1,72 \\
\hline Celtis iguanaea & 1 & 0,30 & & & & & 1 & 0,30 \\
\hline Cinnamomum amoenum & & & 1 & 0,10 & & & 1 & 0,10 \\
\hline Cinnamomum glaziovii & & & & & 8 & 1,68 & 8 & 1,68 \\
\hline Citronella gongonha & & & & & 2 & 0,63 & 2 & 0,63 \\
\hline Cryptocarya aschersoniania & 7 & 0,40 & 34 & 0,78 & 7 & 0,46 & 48 & 0,55 \\
\hline Cryptocarya moschata & & & & & 1 & 0,72 & 1 & 0,72 \\
\hline Cupania vernalis & & & 2 & 2,11 & 1 & 1,28 & 3 & 1,70 \\
\hline Dasyphyllum spinescens & & & & & 4 & 1,21 & 4 & 1,21 \\
\hline Dasyphyllum tomentosum & & & 1 & 0,22 & & & 1 & 0,22 \\
\hline Dicksonia sellowiana & & & 8 & 1,13 & & & 8 & 1,13 \\
\hline Eugenia involucrata & 1 & 0,57 & 1 & 0,70 & & & 2 & 0,64 \\
\hline Eugenia psidiiflora & 21 & 0,39 & 13 & 0,30 & 1 & 0,30 & 35 & 0,33 \\
\hline Eugenia uruguayensis & 11 & 0,27 & 8 & 0,57 & 2 & 0,60 & 21 & 0,48 \\
\hline Gordonia acutifólia & 1 & 1,20 & 2 & 1,06 & & & 3 & 1,13 \\
\hline Ilex brevicuspis & 3 & 1,27 & 19 & 1,17 & 12 & 1,02 & 34 & 1,15 \\
\hline Ilex dumosa & 2 & 1,50 & & & 11 & 0,64 & 13 & 1,07 \\
\hline Ilex paraguariensis & 1 & 0,42 & 15 & 1,00 & 17 & 0,60 & 33 & 0,67 \\
\hline Inga Vera & & & 6 & 0,21 & 7 & 0,58 & 13 & 0,40 \\
\hline Lamonia ternata & & & 8 & 0,67 & 6 & 0,88 & 14 & 0,78 \\
\hline Lithraea brasiliensis & & & 2 & 0,25 & 1 & 0,19 & 3 & 0,22 \\
\hline Lonchocarpus campestris & & & & & 6 & 0,51 & 6 & 0,51 \\
\hline Luehea divaricata & & & & & 12 & 1,15 & 12 & 1,15 \\
\hline Matayba elaeagnoides & 1 & 0,80 & 2 & 1,95 & 10 & 1,14 & 13 & 1,30 \\
\hline Maytenus evonymoides & & & & & 3 & 1,32 & 3 & 1,32 \\
\hline Myrceugenia cucullata & 2 & 0,35 & 7 & 0,59 & 4 & 0,52 & 13 & 0,49 \\
\hline Myrceugenia miersiana & 3 & 0,19 & 3 & 0,12 & & & 6 & 0,16 \\
\hline Myrcia oligantha & 8 & 0,19 & 5 & 0,27 & & & 13 & 0,23 \\
\hline Myrcianthes gigantea & 2 & 0,61 & 1 & 0,50 & 1 & 0,52 & 4 & 0,54 \\
\hline Myrcianthes pungens & & & & & 1 & 0,15 & 1 & 0,15 \\
\hline Myrciaria floribunda & 3 & 0,41 & 3 & 0,59 & & & 6 & 0,50 \\
\hline Myrrhinium atropurpureum & & & 3 & 0,35 & & & 3 & 0,35 \\
\hline Myrsine sp. & & & 3 & 0,84 & 3 & 0,65 & 6 & 0,75 \\
\hline Myrsine umbelata & 1 & 0,45 & 5 & 0,62 & & & 6 & 0,51 \\
\hline Nectandra megapotamica & 1 & 1,60 & 20 & 1,43 & 2 & 1,55 & 23 & 1,53 \\
\hline
\end{tabular}


TABELA 2: Continuação ...

TABLE 2: Continued ...

\begin{tabular}{|c|c|c|c|c|c|c|c|c|}
\hline \multirow[t]{2}{*}{ Espécie } & \multicolumn{2}{|c|}{ Grupo 1} & \multicolumn{2}{|c|}{ Grupo 2} & \multicolumn{2}{|c|}{ Grupo 3} & \multicolumn{2}{|c|}{ Total } \\
\hline & $\mathrm{N}$ & I & $\mathrm{N}$ & I & $\mathrm{N}$ & I & $\mathrm{N}$ & I \\
\hline Ocotea indecora & 4 & 0,86 & 1 & 0,38 & & & 5 & 0,62 \\
\hline Ocotea puberula & & & 6 & 1,08 & 1 & 0,33 & 7 & 0,71 \\
\hline Ocotea pulchella & 14 & 0,81 & 4 & 1,20 & 5 & 0,42 & 23 & 0,81 \\
\hline Pilocarpus pennatifolius & & & & & 1 & 0,28 & 1 & 0,28 \\
\hline Podocarpus lambertii & & & 15 & 0,41 & & & 15 & 0,41 \\
\hline Prunus myrtifolia & 1 & 0,40 & 3 & 1,62 & 3 & 0,80 & 7 & 0,94 \\
\hline Rollinia rugulosa & 1 & 0,10 & & & 1 & 0,20 & 2 & 0,15 \\
\hline Roupala brasiliensis & 5 & 0,78 & 1 & 0,55 & 1 & 0,35 & 7 & 0,56 \\
\hline Sapium glanutum & 2 & 1,20 & 23 & 0,59 & 1 & 0,75 & 26 & 0,85 \\
\hline Scutia buxifolia & 4 & 0,35 & 1 & 0,25 & 1 & 0,20 & 6 & 0,27 \\
\hline Sebastiania brasiliensis & 7 & 0,48 & 22 & 0,47 & 6 & 0,33 & 35 & 0,43 \\
\hline Sebastiania commersoniania & 24 & 0,57 & 2 & 0,46 & 4 & 0,75 & 30 & 0,59 \\
\hline Seguieria aculeata & 3 & 0,32 & & & & & 3 & 0,32 \\
\hline Siphoneugena reitzii & 8 & 0,60 & 8 & 0,44 & & & 16 & 0,52 \\
\hline Solanum mauritianum & & & 1 & 1,17 & & & 1 & 1,17 \\
\hline Solanum sanctae-catharinae & & & 1 & 0,40 & & & 1 & 0,40 \\
\hline Symplocos tetrandra & & & 1 & 0,78 & & & 1 & 0,78 \\
\hline Symplocos uniflora & & & 2 & 0,31 & & & 2 & 0,31 \\
\hline Vernonia discolor & & & 10 & 1,20 & 1 & 0,60 & 11 & 0,90 \\
\hline Xylosma prockia & & & 1 & 0,22 & & & 1 & 0,22 \\
\hline Xylosma pseudosalzmannii & & & 1 & 0,65 & 3 & 0,40 & 4 & 0,53 \\
\hline Zanthoxylum petiolare & & & & & 1 & 1,09 & 1 & 1,09 \\
\hline Zanthoxylum rhoifolium & & & 1 & 1,42 & 2 & 0,25 & 3 & 0,84 \\
\hline Cipós & 1 & 0,30 & 5 & 0,46 & & & 6 & 0,38 \\
\hline Total & 163 & 21,39 & 337 & 35,20 & 304 & 31,87 & 804 & \\
\hline Incremento médio & & 0,55 & & 0,74 & & 0,70 & & 0,67 \\
\hline
\end{tabular}

Em que: $\mathrm{N}$ = Número de indivíduos; I = I incremento médio anual em diâmetro $(\mathrm{cm})$.

No Grupo 2, que corresponde à vegetação em estágio secundário de sucessão, predominam as espécies Cryptocarya aschersoniana, Sapium glandulatum, Sebastiania brasiliensis, Nectandra megapotamica, Ilex brevicuspis, Podocarpus lambertii, Vernonia discolor e Campomanesia xanthocarpa, além de Campomanesia rhombea, Lamanonia ternata, Casearia decandra, Ilex paraguariensis, Inga vera, Myrceugenia cuculata e Ocotea puberula. Foram exclusivas deste grupo às espécies Acca sellowiana, Cinnamomum amoenum, Dasyphyllum tomentosum, Dicksonia sellowiana, Myrrhynium atropurpureum, Podocarpus lambertii, Solanum mauritianum, Solanum sanctae-catharinae, Symplocos tetandra, Symplocos uniflora e Xylosma prockia, a maioria delas características de formações secundárias.

No Grupo 3, a mata primária típica da Floresta Ombrófila Mista, Araucaria angustifolia, Casearia decandra, Blepharocalyx salicifolius, Ilex paraguariensis, Luehea divaricata, Ilex dumosa, Matayba elaeagnoides e Cunnamomum glaziovi foram as espécies predominantes. Foram exclusivas deste grupo às espécies Banara parviflora, Casearia obliqua, Cedrela fissilis, Cinnamomum glaziovii, Citronella gonconha, Chryptocarya moschata, Dasyphyllum spinescens, Longhicarpus campestris, Luehea divaricata, Maytenus evonymoides, Myrcianthes pungens, Pilocarpus pennatifolius e Zanthoxylum petiolare.

Com relação ao crescimento, observa-se que o Grupo 2 (Floresta secundária) apresentou maior incremento médio anual em diâmetro, normal por apresentar espécies pioneiras que apresentam como características maior crescimento, seguido do Grupo 3 (Floresta primária) e do Grupo 1 (Floresta de áreas 
úmidas).

Observa-se, pela Tabela 2, que as espécies Gorndonia acutifolia, Ilex brevicuspis, Matayba elaeagnoides e Nectandra megapotamica apresentaram maiores incrementos anuais em diâmetros em todos os grupos encontrados.

No Grupo 1, Ilex dumosa, Nectandra megapotamica, Ilex brevicuspis, Gordonia acutifolia, Sapium glandulatum, Blepharocalyx salicifolius, Ocotea indecora, Ocotea pulchella, Matayba elaeagnoides e Roupala brasiliensis foram as espécies de maiores incrementos médios anuais em diâmetro.

No Grupo 2, apresentaram maior incremento as espécies Cupania vernalis, Matayba elaeagnoides, Prunus myrtifolia, Nectandra megapotamica, Ocotea pulchella, Vernonia discolor, Ilex brevicuspis, Solanum mauritianum, Dicksonia sellowiana, Ocotea puberula, Gordonia acutifolia, Ilex paraguariensis, Blepharocalix salicifolius, Capomanesia xanthocarpa, Cryptocarya aschersoniana e Symplocos tetandra.

No Grupo 3, foram as espécies Cedrela fissilis, Cinnamomum glaziovii, Nectandra megapotamica, Maytenus evonymoides, Cupania vernalis, Dasyphyllum spinescens, Luehea divaricata, Matayba elaeagnoides, Zanthoxylum petiolare, Ilex brevicuspis, Banara parviflora e Prunus myrtifolia.

No geral, Cedrela fissilis, Cupania vernalis, Cinnamomum glaziovii, Nectandra megapotamica, Maytenus evonymoides, Matayba elaeagnoides, Dasyphylum spinescens, Solanum mauritianum, Ilex brevicuspis, Luehea divaricata, Dicksonia sellowiana, Gordonia acutifólia, Znthoxylum petiolare, Ilex dumosa, Prunus myrtifolia, Banara parviflora, Vernonia discolor, Sapium glandulatum, Zanthoxylum rhoifolium, Ocotea puberula, Casearia obliqua, Blepharocalyx salicifolius, Lamanonia ternata, Symplocos tetandra, Cryptocarya moschata, Campomanesia xanthocarpa, Ilex paraguariensis e Ocotea indecora foram às espécies que apresentaram maiores incrementos em diâmetro. Contudo, deve-se ressaltar que certas espécies tiveram poucos ou um único indivíduo amostrados.

\section{CONCLUSÕES}

Com os resultados obtidos no trabalho conclui-se que:

1) Foi possível diferenciar na Floresta Ombrófila Mista da FLONA de São Francisco de Paula, três grupos florísticos: Floresta de áreas úmidas, Floresta secundária e Floresta primária.

2) O Grupo 1 apresenta menor diversidade florística e menor incremento em diâmetro. Tem as espécies Sebastiania commersoniana, Eugenia psidiiflora, Ocotea pulchella, Eugenia uruguayensis e Myrcia oligantha como as mais características.

3) O Grupo 2 tem o maior incremento em diâmetro e apresenta as espécies Cryptocarya aschersoniana, Sapium glandulatum, Sebastiania brasiliensis, Nectandra megapotamica, Ilex brevicuspis, Podocarpus lambertii, Vernonia discolor e Campomanesia xanthocarpa como as mais representativas.

4) O Grupo 3 é o grupo típico da Floresta Ombrófila Mista e tem como mais características as espécies Araucaria angustifolia, Casearia decandra, Blepharocalyx salicifolius, Ilex paraguariensis, Luehea divaricata, Ilex dumosa, Matayba elaeagnoides e Cunnamomum glaziovi.

5) Em programas de manejo florestal recomenda-se dar ênfase ao cultivo das espécies Araucaria angustifolia, Cedrela fissilis, Cupania vernalis, Cinnamomum glaziovii, Nectandra megapotamica, Matayba elaeagnoides, Ilex brevicuspis, Luehea divaricata, Dicksonia sellowiana, Prunus myrtifolia, Ocotea puberula, Blepharocalyx salicifolius, Lamanonia ternata, Cryptocarya moschata, Ilex paraguariensis e Ocotea indecora, por apresentarem maiores incrementos médios anuais em diâmetro e possuírem potencialidades econômica

\section{REFERÊNCIAS BIBLIOGRÁFICAS}

ARAUJO, M. M. Vegetação e mecanismos de regeneração em fragmento de Floresta Estacional Decidual Ripária, Cachoeira do Sul, RS, Brasil. Santa Maria, 2002.153f. Tese (Doutorado em Engenharia Florestal) Universidade Federal de Santa Maria, Santa Maria, 2002.

BUDOWSKI, G. Distribution of tropical american rain forest species in the light of sucessional processes. Turrialba, v. 15, n. 1, p. 40-42, 1965.

DAUBENMIRE, R. Plant communities : a textbook of plant synecology. New York : Harper \& Row, 1968. 300p. 
GANDOLFI, S. Estudo florístico e fitossociológico de uma floresta residual na área do Aeroporto Internacional de São Paulo, município de Guarulhos, SP. Campinas, 1991. 232f. Dissertação (Mestrado em Biologia Vegetal) Instituto de Biologia, Universidade Estadual de Campinas, Campinas, 1991.

GÓMEZ-POMPA, A., WIECHERS, B. L. Regeneración de los ecosistemas tropicales y subtropicales. In: GOMÉZPOMPA, A. et al. (Eds.). Investigaciones sobre la regeneración de selvas altas en Veracruz, México. México: Continental, 1976. p. 11-30.

HILL, M. O. Twinspan: a FORTRAN program to arranging multivariate data in na ordered two way table by classification of individual and attributes. Ithaca, New York: Cornell University, 1979. 60p.

HILL, M. O.; BUNCE, R. G. H.; SHAW, M. W. Indicator species analysis, a divisive polythetic method of classification, and its application to a survey of native pinewoods in Scotland. Journal of Ecology, v.63, n.2, p.597613, 1975.

HORN, H. S. The ecology of secondary sucession. Ann. Rev. Ecol. Syst., v.5, p.25-37, 1974.

JARENKOW, J. A. Composição florística e estrutura da mata com Araucária na Estação Ecológica de Aracuri, Esmeralda, Rio Grande do Sul. Porto Alegre, 1985. 86f. Dissertação (Mestrado em Ciências Biológicas) Universidade Federal do Rio Grande do Sul, Porto Alegre, 1985.

KAGEYAMA, P. Y., BRITO, M. A., BAPTISTON, I. C. Estudo do mecanismo de reprodução de espécies da mata natural. In: KAGEYAMA, P. Y. (Coord.). Estudo para implantação de matas ciliares de proteção na bacia hidrográfica do Passa Cinco, Piracicaba, SP. Piracicaba: DAEE/USP/FEALQ, 1986. 236p.

KENT, M.; COKER, P. Vegetation description and analysis. London: Behaven Press, 1992. 362 p.

KLEIN, R. M. Ecologia da flora e vegetação do Vale do Itajaí. Sellowia, Itajaí, v.31, 1979.

KLEIN, R. M. Ecologia da flora e vegetação do Vale do Itajaí. Sellowia, Itajaí, v.32, 1980.

KUNIYOSHI, Y. S. Reconhecimento de fases sucessionais de vegetação. In: SEMINÁRIO SOBRE AVALIAÇÃO E RELATÓRIO DE IMPACTO AMBIENTAL, 1989, Curitiba. Anais... Curitiba: FUPEF, 1989. p. 97-107.

LONGHI, S. J. Agrupamento e análise fitossociológica de comunidades florestais na sub-bacia hidrográfica do rio Passo Fundo-RS. Curitiba, 1997. 193f. Tese (Doutorado em Ciências Florestais) - Universidade Federal do Paraná, Setor de Ciências Agrárias, Curitiba, 1997.

MORENO, J. A. Clima do Rio Grande do Sul. Porto Alegre : Governo do Estado do Rio Grande do Sul, Secretaria da Agricultura, Diretoria de Terra e Colonização, 1961. 42 p.

NARVAES, I. S. Classificação e caracterização da regeneração natural em floresta ombrófila mista na Floresta Nacional de São Francisco de Paula, RS. Santa Maria, 2004. 143f. Dissertação (Mestrado em Engenharia Florestal) Universidade Federal de Santa Maria, Santa Maria, 2004.

ODUM, E. P. Fundamentos da ecologia. 4. ed. Lisboa: Fundação Calouste Gulbenkian, 1988. 927p.

RIO GRANDE DO SUL Secretaria Estadual do Meio Ambiente. Inventário florestal contínuo do Rio Grande do Sul. Santa Maria: SEMA/UFSM, 2002. 706p. (Relatório).

RODRIGUES, R. R. A sucessão florestal. In: MORELlATO, P. C., LEITÃo FILHO, H. F. (Orgs.). Ecologia e preservação de uma floresta tropical urbana: Reserva de Santa Genebra. Campinas : UNICAMP, 1995. p. 30-36.

$136 \mathrm{p}$.

VACCARO, S. Caracterização fitossociológica de três fases sucessionais de uma floresta estacional decidual, no município de Santa Tereza - RS. Santa Maria, 1997. 92f. Dissertação (Mestrado em Engenharia Florestal) - Centro de Ciências Rurais, Universidade Federal de Santa Maria, Santa Maria, 1997.

WHITMORE, T. C. Canopy gaps and two major groups of forest trees. Ecology, v. 70, n. 3, p. 536-538, 1989. 